\title{
Context-aware Life-Logging for Persons with Mild Dementia
}

\author{
Basel Kikhia, Josef Hallberg, Kåre Synnes, and Zaheer ul Hussain Sani
}

\begin{abstract}
The demands of introducing technology to support independent living is increasing. This is true also for persons suffering from mild dementia who may have difficulties remembering important information, such as activities, numbers, names, objects, faces, and so on. This paper presents a context-aware life-logging system, called MemoryLane, which can support independent living and improve quality of life for persons with mild dementia. The system offers both real time support as well as possibilities to rehearse and recall activities for building episodic memory. This paper also presents a mobile client to be used in MemoryLane, as well as an evaluation of the importance of different data for the purpose of memory recollection.
\end{abstract}

\section{INTRODUCTION}

Persons with mild dementia have difficulties remembering daily activities, remembering names, objects, phone numbers, faces, and so on; they often rely on outside memory aids to help them compensate for their memory deficits [1]. Studies have shown that retrospective memory aids can help persons with mild dementia rehabilitate their memories [2]. Therefore, significant support can be provided by a system which offers context-aware services in combination with life-logging aspects, such as recording data about daily activities and making it available for later retrieval.

This paper presents a context-aware life-logging system for persons with mild dementia, called MemoryLane, which offers real time support (navigation, nearby person identification, current location identification, activity guide, etc) and possibilities to review previous activities and to organize future ones. The purpose of this system is to increase the person's sense of safety and to help the person build episodic memory.

The target group, persons with mild dementia, may have

Manuscript received April 6, 2009. This work is co-funded by the European Regional Development Fund (Mål-2) through the Centre for Distance-spanning Health-care (CDH)

Basel Kikhia is a PhD student in the department of Computer Science and Electrical Engineering, Luleå University of Technology, Sweden. 971 87 Luleå, Sweden. phone: +46-920-491000; fax: +46-920-491399; e-mail: Basel.Kikhia@ltu.se.

Josef Hallberg is a $\mathrm{PhD}$ in the department of Computer Science and Electrical Engineering, Luleå University of Technology, Sweden. 97187 Luleå, Sweden. phone: +46-920-491000; fax: +46-920-491399; e-mail: Josef.hallberg@ltu.se.

Kåre Synnes is a $\mathrm{PhD}$ in the department of Computer Science and Electrical Engineering, Luleå University of Technology, Sweden. 97187 Luleå, Sweden. phone: +46-920-491000; fax: +46-920-491399; e-mail: Kare.Synnes@1tu.se.

Zaheer ul Hussain Sani is a developer in the MemoryLane project, Lulea University of Technology, Sweden. 97187 Luleå, Sweden. phone: +46920-491000; fax: +46-920-491399; e-mail: zaheer@memorylane.nu. problems operating advanced technology because of the difficulty remembering the operation procedures. It is therefore important to consider the entire operation flow, which tasks should be handled by the person with mild dementia alone, and which tasks should be handled with the assistance of a carer. Moreover, it is important to collect enough data to provide sufficient material for reviewing the activities at the end of the day. This may be problematic outside the home since the system has to rely on data collected by equipments carried by the person with mild dementia. However, inside the home the system can collect data from the sensorized environment. This paper presents an investigation of some of these problems and answers the following questions:

1) How could the operation and data flow be designed to help persons with mild dementia review their life in a digital format?

2) How important is the data that can be collected by portable devices for persons with mild dementia?

The rest of this article is organized as follows. The second section presents related work. The third section describes a scenario which presents the functionality of the proposed system. The architecture and operation flow is presented in the fourth section, and the fifth section presents the current implantation of the mobile client. The evaluation of the importance of collected data is presented in section six and section seven discusses the results and research questions. Finally the eighth section concludes the paper and presents future work.

\section{RELATED WORK}

There are recent research on logging the life of persons and recording it in a digital format. Furthermore, many applications have been developed in the field of smart environments especially for elderly persons and persons with mild dementia to improve their quality of life.

Researchers in Lifestyle Assistant [3] and Cogknow [5] aim to build a context-aware system that can be used by elderly persons and persons with mild dementia. The purpose is to help them live independently longer in their homes and to increase the safety in their life. Implementing such systems inside the home of a person will convert it into an intelligent space that observes the user and assists with daily activities. For instance, the system will remind the user to do daily activities, and it will send warning signals if necessary (forgetting the food on the stove, forgetting the door open, etc). Some efforts have also been directed 
towards implementing friendly systems which can support and improve the user's social life (Persona [6] and Netcarity [9]).

Other studies such as Context-Coded Memories [10] and MyLifeBits [7] are targeting life-logging aspect by recording the life of a person in a digital format and make it available for later retrieval. The complexity of such systems varies depending on the used recording devices and the provided applications for recalling. For instance, the mobile device is the main tool for recording data in PENSIEVE project [8], while extra devices like cameras have been proposed in other studies to be used in a life-logging system. Furthermore, O'Connor et al. [4] implemented low level analysis for the collected images during the day to improve the classification and the retrieval task in their target lifelogging system (MediAssist).

Using life-logging aspects to support memory of persons with mild dementia is an ongoing process and a challenge since the target users might have difficulties in dealing with technology.

\section{SCENARIO}

This section describes a scenario featuring Lisa who is a woman suffering from mild dementia. She has been provided with the MemoryLane system to support her. The scenario describes in which ways the MemoryLane system can help her throughout the day, and how to use the system together with a carer to review the day and possibly build episodic memory.

Lisa is on her way to the medical centre for her medical check-up and she is following the navigation instructions given to her by the mobile device, which is running the MemoryLane mobile client, to get there. She is grateful for this device because it helps her remember past activities, places, and name of persons. Today she is meeting with Dr. Stefan, and as she sits down in the waiting room she is reminded of his name by the mobile device that has detected the doctor's presence. While she is waiting she takes the opportunity to review and recall past activities where Dr. Stefan has been present.

At the end of the day, when Lisa is back in her home and her carer is with her, Lisa sits down with her carer to review the activities of the day. The data from the portable devices is uploaded to the home station and they can start reviewing. Together they select a number of images that represent the different activities: a few images of the clinic, an image from the laundry room from when Lisa was doing her laundry, and images from the dinner Lisa had with her friends. Some images and activities are already proposed by the system, based on various sensor data, and the names of known persons which were present during the activities are automatically added by the system to reduce the effort needed to review the activities. Once they are done with the review process, the carer helps Lisa add "Your son is visiting on Friday" as a future activity so she will not forget.
The previous scenario described how the MemoryLane application provided Lisa with real-time support, such as navigating to the doctor's office and reminding her about her doctor's name and past activities with him. The scenario also describes the review process where Lisa can review and rehearse the activities she has done during the day. In the reviewing process, she is assisted by her carer who can help in naming activities and in adding details which are not automatically detected by the system. Reviewing activities in this way helps in building episodic memory, and it provides the possibility of recalling past activities on the home station or the mobile device at a later time. Finally, the scenario shows that future activities can be added to the system for reminder purposes.

\section{ARCHITECTURE}

The authors combine results from previous context-aware systems in a tool that can assist a person with mild dementia, indoors and outdoors. The authors extend the previous works in life-logging as well by introducing a review client at home which will be a calendar for the person with mild dementia that can visualize the life as lists of activities. Moreover, reducing the intervention from the user side in naming the collected data and improving the usability of the system is a main goal for the target system since it may be difficult for persons with mild dementia to operate advanced technology.

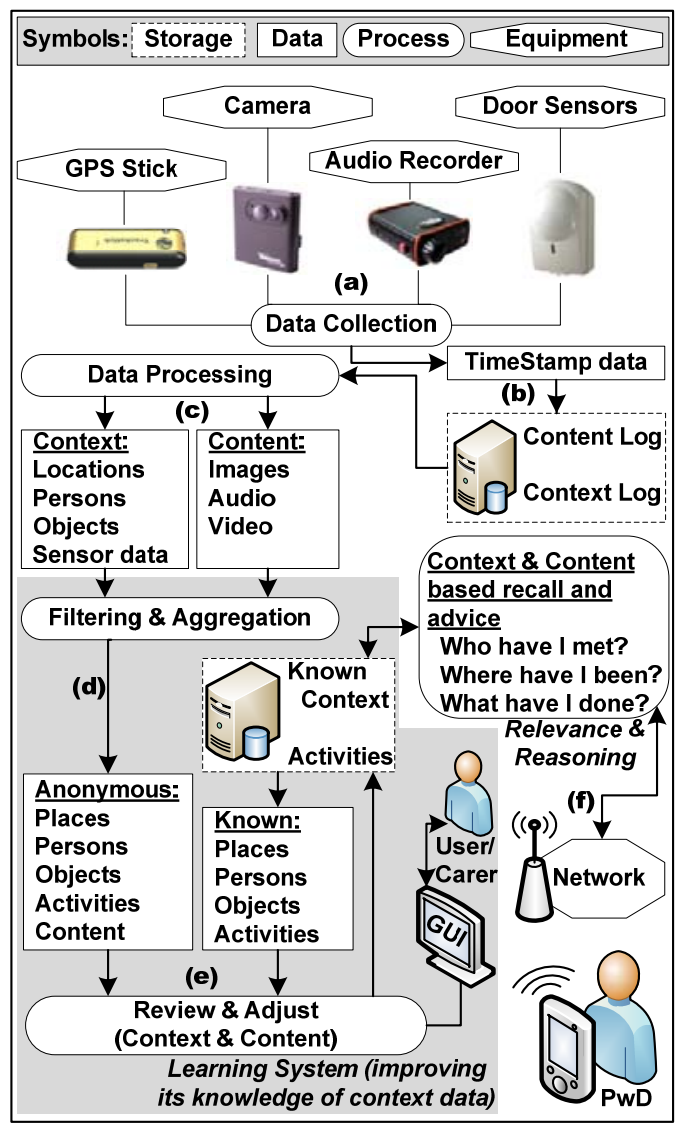

Fig. 1. The MemoryLane architecture 
Fig. 1 shows how the data and operation flow can be designed to create a usable system for a person with mild dementia where advanced parts are assisted by a carer. The different steps have been marked by a letter to clarify the sequence of the data.

Sensor data, such as images and GPS data, is collected automatically by devices either carried by the person, or located in the environment (a). This step requires the person with mild dementia to bring the relevant equipments and have them turned on. The data will then be time-stamped and transferred to a local storage in the system (b). Time is an important factor when trying to automatically sort data into different activities. When data has been uploaded, it is processed (c) and divided into context data (persons, places, and objects) and content data (images, audio, and video), and then, it will be analyzed, filtered and aggregated automatically by the system (d).

After the data has been filtered and aggregated, it is time for the person with mild dementia together with the carer to review and adjust the context and content data (e). The carer can assist the person with mild dementia by organizing images, giving titles to activities, naming places (if not already named by the system), and naming persons appearing in the activities (if not already named by the system). At this stage, the person with mild dementia also has the opportunity to rehearse, with the assistance of the carer, the activities done that day to build episodic memory.

New context data, generated during the review process, is stored and used in future review sessions as well as in the mobile device, so the system can give suggestions to ease the review process. The last step in the data flow is to provide the known context data with their related content data as lists of activities through a graphical user interface (f), so the person with mild dementia can get support and recall activities.

\section{THE MOBILE CLIENT}

The aim of the mobile client is to provide an application on the mobile device which can support the person with mild dementia during the day. Initially two main functionalities have been implemented: nearby person detection, and current location detection. Detecting the presence of known persons nearby is based on the Bluetooth ID, and the interface displays images of up to four persons if there are more than one known person nearby. Clicking on the persons' image will show the images of all nearby known persons separately as a list, making it easier for the person with mild dementia to choose any person to recall information about. Detecting the current location of the person with mild dementia is based on GPS coordinates, and clicking on the location icon will display information relevant to that location such as the name, the address, etc.

Fig. 2 shows the main interface of the mobile client. In this figure, the left bottom corner displays the nearby known persons, while the right bottom one shows the image and the name of the current location. The left upper part shows the current time, while the right upper one presents the next activity that the person with mild dementia has based on the review client at home.

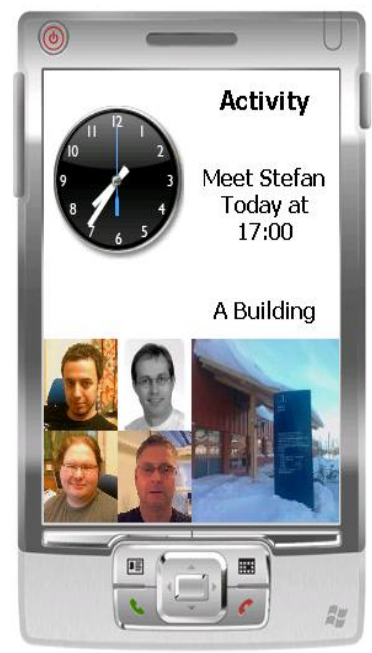

Fig. 2. The main interface of the mobile client

\section{Evaluation}

Portable devices such as smart-phones are able to collect a number of different context and content data as well as to present real-time support tools. To evaluate the importance of data that is possible to be collected by portable devices for memory recollection and the usefulness of simple realtime support tools, we asked 7 persons to fill a questionnaire. Three of the persons are healthcare professionals who are not technology experts but work close to persons with mild dementia, while the rest are working in the domain of assistive technology for elderly persons and persons with mild dementia.

The questions in the questionnaire were related to location indication, nearby persons, nearby objects, images, audio recording, logging calls, showing next activity, and picture dialing. Each question had a rank from 1 to 10 where 1 means that this kind of data is not important and 10 means that this data is very important for memory recollection. A presentation about MemoryLane and a demonstration of the mobile client were given to the audience before asking them to fill the questionnaire. Table I shows the outcome of the questionnaire.

The answers given in the questionnaire by the participants differed, and we chose to calculate the average of the results to get an approximate number that can indicate the opinions of the experts and healthcare professionals. According to the questionnaire, images have the highest average as 9.1, while logging calls has the lowest one as 6.1; other kinds of data vary in average between 7.2 and 8.8 (Table I). These results indicate that the proposed data collection, with a possible exception for the call log, is important for memory recollection. 
TABLE I

IMPORTANCE OF THE COLLECTED DATA

\begin{tabular}{|c|c|c|c|c|c|c|c|c|}
\hline \multirow[b]{2}{*}{ Users } & \multicolumn{3}{|c|}{$\begin{array}{c}\text { Healthcare } \\
\text { Professionals }\end{array}$} & \multicolumn{4}{|c|}{ Experts } & \multirow{2}{*}{ Avg } \\
\hline & 1 & 2 & 3 & 4 & 5 & 6 & 7 & \\
\hline $\begin{array}{l}\text { Location } \\
\text { indication }\end{array}$ & 10 & 6 & 8 & 8 & 7 & 9 & 8 & 8 \\
\hline $\begin{array}{l}\text { Nearby } \\
\text { persons }\end{array}$ & 10 & 10 & 10 & 5 & 6 & 10 & 9 & 8.5 \\
\hline $\begin{array}{l}\text { Nearby } \\
\text { objects }\end{array}$ & 10 & 7 & 6 & 7 & 10 & 5 & 6 & 7.2 \\
\hline Images & 10 & 10 & 10 & 10 & 7 & 8 & 9 & 9.1 \\
\hline $\begin{array}{c}\text { Audio } \\
\text { recording }\end{array}$ & 10 & 7 & 6 & 7 & 7 & 5 & 9 & 7.2 \\
\hline $\begin{array}{l}\text { Logging } \\
\text { calls }\end{array}$ & 5 & 6 & 7 & 8 & 8 & 4 & 5 & 6.1 \\
\hline $\begin{array}{c}\text { Showing } \\
\text { next activity }\end{array}$ & 10 & 8 & 8 & 9 & 10 & 9 & 8 & 8.8 \\
\hline $\begin{array}{l}\text { Picture } \\
\text { dialing }\end{array}$ & 7 & 8 & 10 & 8 & 6 & 8 & 8 & 7.8 \\
\hline
\end{tabular}

\section{DISCUSSION}

In section I, two questions were asked. The first question "How could the operation and data flow be designed to help persons with mild dementia review their life in a digital format?" has been partially answered by MemoryLane architecture (Fig. 1). The data and operation flow has been designed to not be complicated for the person with mild dementia. The gathering of data only requires the person with mild dementia to carry the portable devices and make sure they are turned on. When reviewing the activities of the day the person with mild dementia is assisted by the carer.

The carer can help in navigating the user interface as well as in filling some information the person with mild dementia may have forgotten. Together they can rehearse the activities done that day in order to help build episodic memory. The review process results in a list of activities that can be later recalled by the person with mild dementia for further rehearsal.

The second question "How important is the data that can be collected by portable devices for persons with mild dementia?" has been partially answered by the questionnaire (Table I). Only seven persons were asked so the questionnaire does not provide statistical significance, but it gives an indication of importance to be used for future development. Furthermore, the questionnaire does not say if the proposed data collection is sufficient for determining activities and assisting with building episodic memory. This is something which needs to be evaluated by developing the system and letting persons with mild dementia use it.

\section{CONCLUSION \& FUTURE WORK}

Combing context-awareness and life-logging aspects in one system can provide support for persons with mild dementia. In this paper, we introduced a context-aware lifelogging system that can support a person with mild dementia by providing real-time services and the possibility to recall past activities and plan future ones. In addition, we presented the main interface of the mobile client within the system. The evaluation showed that different kinds of data have different impacts on memory recollection for a person with mild dementia, so we will prioritize functions in our future work based on the importance of data. The next stage in our work is to continue the development of the mobile client and the review client of MemoryLane, so we can further test the feasibility of those clients with persons with mild dementia.

\section{ACKNOWLEDGMENT}

We would like to extend our gratitude to Johan Bengtsson and Stefan Kullberg for their ideas and support.

\section{REFERENCES}

[1] Lee, M.L. \& Dey, A.K. Capturing and Reviewing Context in Memory Aids. In: Workshop on Designing Technology for People with Cognitive Impairments, 2006.

[2] Hodges. S, Williams. L, Berry. E, Izadi. S, Srinivasan. J, Butler. A, Smyth. G, Kapur. N, \& Wood. K. SenseCam: A Retrospective Memory Aid. Microsoft Research, 2006.

[3] K. Z. Haigh, C. W. Geib, C. A. Miller, J. Phelps, and T. Wagner. Agents for recognizing and responding to the behaviour of an elder. In AAAI Workshop on Automation as Caregiver: The Role of Intelligent Technology in Elder Care, pp 31-38, Canada, 2002.

[4] Noel E. O'Connor, Edward Cooke, Herve Le Borgne, Michael Blighe, Tomasz Adamek. The Acetoolbox: Low-Level Audiovisual Feature Extraction for Retrieval and Classification. In: Integration of Knowledge, Semantics and Digital Media Technology, EWIMT, 2005.

[5] Meiland, F.J.M., Reinersmann, Bergvall-Kareborn, B., Graig, D., Moelaert, F., Mulvenna, M.D., Nugent, C., Scully, T., Bengtsson, J.E., Droes, R.M. CogKnow: Development of an ICT device to support people with dementia. Journal of Information Technology in Healthcare 5(5), 324-334, 2007.

[6] Persona project. http://www.aal-persona.org/deliverables.html, March 2009

[7] Gemmell J, Bell G, Lueder R, Drucker S, \& Wong C. MyLifeBits: Fulfilling the Memex Vision. Microsoft Research, Juan-les-Pins, France, pp. 235-238, 2002.

[8] Technology to Aid Human Memory, IBM Research, http://www03.ibm.com/press/us/en/pressrelease/24750.wss, March 2009.

[9] A. Cappelletti, B. Lepri, N. Mana, F. Pianesi, and M. Zancanaro. A multimodal data collection of daily activities in a real instrumented apartment. In: Workshop on Multimodal Corpora. Marrakech, Morocco, 2008.

[10] Bisht M., Swords D., Quigley A., Gaudin B. and Bennett M.. ContextCoded Memories: "Who, What, Where, When, Why?: Supporting Human Memory with Interactive Systems. Workshop at the British HCI International Conference, 2007. 